\title{
Análisis multinivel de variables relacionadas con el paciente, el diente y la localización en el diente sobre la profundidad de la bolsa periodontal en pacientes con periodontitis crónica ${ }^{1}$
}

\section{A multilevel approach of patient, tooth and tooth site -related variables on the probing depth in patients with chronic periodontitis}

\author{
ARDILA MEDINA CM* \\ VERGARA $S * *$ \\ GUZMÁN ZULUAGA IC***
}

\begin{abstract}
Ardila Medina CM, Vergara S, Guzmán Zuluaga IC. Análisis multinivel de variables relacionadas con el paciente, el diente y la localización en el del diente sobre la profundidad de la bolsa periodontal en pacientes con periodontitis crónica. Av Periodon Implantol. 2013; 25, 2: 91-97.
\end{abstract}

\section{RESUIMEN}

Introducción: El análisis multinivel permite considerar el comportamiento de medidas repetidas de localizaciones individuales, conociendo que están agrupadas en dientes y anidadas dentro de sujetos. Objetivo: evaluar la relativa contribución de factores relacionados con el sujeto, el diente y la localización en el diente en la profundidad de la bolsa periodontal.

Materiales y métodos: Se examinaron los parámetros clínicos y la presencia de microorganismos subgingivales en 76 pacientes con periodontitis crónica. Mediante un análisis de regresión multinivel, se evaluó la influencia de diferentes factores sobre la profundidad de sondaje.

Resultados y conclusiones: La mayor parte de la varianza se atribuyó al nivel localización en el diente $(63 \%)$, seguido por el nivel diente $(23 \%)$ y el nivel paciente $(14 \%)$. Los fumadores $(p=0,003)$ se asociaron significativamente con bolsas profundas. Los dientes anteriores presentaron menor profundidad de sondaje $(p=0,0002)$. Se observó una mayor profundidad en localizaciones proximales $(p=0,008)$, con sangrado al sondaje $(p=0,0002)$ y presencia de placa bacteriana $(p=0,008)$. El análisis multinivel reveló que el mayor porcentaje de la varianza se asoció con el nivel localización en el diente, mientras el menor se relacionó con el nivel paciente.

PALABRAS CLAVE: Periodontitis crónica, análisis multinivel, bolsas periodontales.

\section{SUIMIMARY}

Introduction: Multilevel analysis allows us to consider the behavior of repeated measurements of individual sites, knowing that teeth are grouped into nested within subjects.

Objective: to assess the relative contribution of factors related to the subject, tooth and tooth site in the periodontal pocket depth.

Materials and Methods: Clinical parameters and subgingival microorganisms were evaluated in 76 patients with chronic periodontitis. The influence of different factors on the probing depth was examined using a multilevel regression analysis.

Results and conclusions: Most of the variance was attributed to site level (63\%), followed by the tooth level $(23 \%)$ and patient level $(14 \%)$. Smokers $(p=0.003)$ were significantly associated with deep pockets. The anterior teeth presented lower probing depth $(p=0.0002)$. The model showed greater

Investigación financiada por la Facultad Nacional de Salud Pública y el Grupo de Epidemiología de la Universidad de Antioquia.

* Profesor asociado. Facultad de Odontología. Universidad de Antioquia. Candidato a Ph.D en Epidemiología. Universidad de Antioquia. Grupo de Epidemiología Universidad de Antioquia.

** Periodoncista. Universidad Javeriana. Profesora de cátedra Universidad de Antioquia.

*** Periodoncista. Universidad de Chile. Profesora asistente. Facultad de Odontología. Universidad de Antioquia. 
probing depth associated with proximal sites $(p=0.008)$, bleeding on probing $(p=0.0002)$ and presence of plaque $(p=0.008)$. Multilevel analysis revealed that the highest percentage of variance associated with the site level, while the lowest level was related to the patient.

KEY WORDS: Chronic periodontitis, multilevel analysis, probing depth.

Fecha de recepción: 11 de junio de 2010.

Fecha de aceptación: 20 de junio de 2010.

\section{INTRODUCCIÓN}

La periodontitis es una infección compleja presente en individuos susceptibles y causada principalmente por una biopelícula formada sobre la superficie de los dientes (1). La reacción inflamatoria e inmune frente a la presencia de la biopelícula es responsable de la pérdida de inserción periodontal y del hueso de soporte (2). Estos procesos destructivos incluyen cambios bioquímicos celulares registrados como eventos clínicos en niveles superiores que pueden variar en diferentes localizaciones de un diente (3). De esta forma, tres niveles pueden estar involucrados en el proceso inflamatorio periodontal: el individuo, el diente y la localización en el diente. El examen periodontal proporciona información de diferentes localizaciones en distintos dientes de los pacientes. La estructura jerárquica inherente a los datos periodontales presenta dificultades para el análisis ya que puede perderse información y sobrestimarse el error estándar, al agregar datos de la localización en el diente dentro de los pacientes, debido a que se utilizan valores promedios. De la misma forma, puede subestimarse el error estándar al realizar análisis en el diente o en el nivel localización del diente sin tener en cuenta la dependencia existente entre dientes o localizaciones en un paciente. El modelo multinivel plantea un enfoque estadístico para superar este problema ya que puede usarse en el análisis del comportamiento de medidas repetidas de localizaciones individuales conociendo que se agrupan en dientes (niveles inferiores) y se anidan dentro de sujetos (nivel superior) (4).

La profundidad de la bolsa periodontal evalúa el grado de reacción del huésped (1) y su relación con el proceso de regulación homeostático huésped-parásito (5). Además, la bolsa periodontal es un signo relevante de la periodontitis debido a que las bolsas profundas favorecen el crecimiento de periodontopatógenos y son un signo de la presencia de reacción inflamatoria (5).

El objetivo de este estudio fue evaluar la relativa contribución de factores relacionados con el sujeto, el diente y la localización en el diente, sobre la profundidad de la bolsa periodontal, utilizando un análisis multinivel.

\section{MATERIALES Y MÉTODOS}

\section{Sujetos}

En este estudio se eligieron 76 sujetos sistémicamente sanos (45 mujeres y 31 hombres) que cumplieron los criterios de selección determinados para la presente investigación. Estos pacientes asistieron a las clínicas odontológicas de la Facultad de Odontología de la Universidad de Antioquia entre octubre de 2008 y marzo de 2009. Cada participante firmó un consentimiento informado. El Comité de Ética de la Sede de Investigación Universitaria de la Universidad de Antioquia aprobó el diseño del estudio teniendo en cuenta la Declaración de Helsinki sobre experimentación que involucra seres humanos. En este estudio participaron pacientes con diagnóstico de periodontitis crónica. Este diagnóstico se realizó según los criterios recomendados por la Academia Americana de Periodoncia (AAP) (6). Los criterios de exclusión fueron embarazo, lactancia, presencia de diabetes o cualquier enfermedad sistémica que alterara el curso de la enfermedad periodontal, terapia periodontal en el último año, y utilización de antimicrobianos o antiinflamatorios no esteroides en los seis meses previos al examen clínico y a la toma de muestras microbiológicas.

\section{Evaluación clínica}

A cada paciente se le realizó una historia clínica, además de un examen clínico y radiográfico completo. La información relacionada con el hábito de fumar (fumador sí/no) se obtuvo mediante interrogatorio. Uno de los autores $(\mathrm{CA})$ realizó todos los exámenes clínicos. En el examen inicial se evaluó la presencia de placa visible $(0 / 1)$, sangrado al sondaje $(0 / 1)$, profundidad de sondaje y nivel de inserción clínica en seis 
localizaciones (mesiobucal, bucal, distobucal, distolingual, lingual y mesiolingual) usando una sonda periodontal calibrada (UNC-15, Hu-Friedy, Chicago, IL). El compromiso de furcación se evaluó según las recomendaciones de Hamp y colaboradores (7), utilizando una sonda Nabers. Antes de iniciar el estudio se evaluó la reproducibilidad intraexaminador (en seis sujetos con al menos 20 dientes) valorando cada uno de los índices, mediante sondaje y examen clínico de la mitad de la boca, en dos oportunidades en la misma semana. Los coeficientes de correlación intraclase para el promedio de la profundidad de sondaje y el nivel de inserción clínica fueron 0,92 y 0,91, respectivamente.

\section{Toma de muestras microbiológicas}

Se tomaron muestras microbiológicas de los pacientes en localizaciones con una profundidad de sondaje $\geq 5 \mathrm{~mm}$. Para la toma de muestras se seleccionaron las seis bolsas periodontales más profundas de cada paciente. Después de aislar la zona con algodón y eliminar la placa supragingival con cureta, se insertaron puntas de papel estéril en cada bolsa periodontal durante 20 segundos. Las muestras de cada paciente se depositaron en $2 \mathrm{~mL}$ de medio de transporte (Viability Medium Göteborg Anaerobically III: VMGA III) (7) y se llevaron al laboratorio de microbiología de la Facultad de Odontología de la Universidad de Antioquía para procesarlas dentro de las dos horas siguientes. Las muestras fueron analizadas utilizando técnicas de cultivo para la presencia de periodontopatógenos de acuerdo a Slots (8). Para la identificación de Porphyromonas gingivalis y Tannerella forsythia se realizaron cinco diluciones en base 10 a partir del medio VMGA III, se sembraron $100 \mu \mathrm{L}$ de las diluciones $10^{-3}, 10^{-4}$ y $10^{-5}$ en agar brucella sangre enriquecido con hemina y menadiona (BBL Microbiology Systems, Cockeysville, MD) y se llevaron a incubación a $36^{\circ} \mathrm{C}$ en atmósfera de anaerobiosis (Anaerogen, Oxoid, Hampshire, England) durante siete días. Para la identificación de Aggregatibacter actinomycetemcomitans, las muestras sin diluir y $10^{-1}$ se sembraron en agar TSBV (tripticasa soya bacitracina vancomicina) y se incubaron en atmósfera de $10 \%$ de $\mathrm{CO}_{2}$ (Campygen, Oxoid, Hampshire, England) durante tres a cinco días. Se realizaron además pruebas bioquímicas y enzimáticas confirmatorias para todos los microorganismos utilizando los sistemas de identificación comercial Rapid ANA II (RemelTM, Apogent) y API ZYM (Biomerieux® Francia). Las cepas presuntivas de $A$. actinomycetemcomitans fueron identificadas por la presencia de una estructura seme- jante a una estrella en el interior de las colonias y las pruebas catalasa positiva y MUG negativa (4Metilumbeliferil-B-D-galactosidasa, para investigar la fermentación de la lactosa).

Para el aislamiento de bacilos entéricos gram negativos se utilizó agar MacConkey para luego incubarse a una atmósfera aeróbica a $37^{\circ} \mathrm{C}$ por $24-48$ horas. Sobre las colonias que crecieron en agar MacConkey se empleó tinción de gram para verificación. En agar Cetrimide, se subcultivaron todas las colonias sospechosas de pertenecer al género Pseudomonas. La identificación primaria de las colonias aisladas se realizó por pruebas bioquímicas manuales como oxidasa, citrato, MIO (Movilidad-Indol-Ornitina),lisina, urea de Christensen, TSI (agar hierro triple azúcar), malonato, DNAsa. En el caso de los microorganismos no fermentadores de la glucosa, se utilizaron pruebas adicionales como la oxidación-fermentación (OF) de glucosa, movilidad en gota pendiente y crecimiento a $42^{\circ} \mathrm{C}$. Se realizaron pruebas bioquímicas y enzimáticas confirmatorias para todos los microorganismos utilizando los sistemas de identificación comercial Rapid ANA II (RemelTM, Apogent) y API ZYM (Biomerieux® Francia).

Después de la incubación se calculó el número total de unidades formadoras de colonias por mililitro de cada muestra a partir de los medios de cultivo. El porcentaje relativo de recuperación de cada microorganismo se calculó a partir del total de unidades formadoras de colonias por mililitro (UFC/ml).

\section{Análisis estadístico}

Para describir las variables relacionadas con los sujetos y los dientes se realizó un análisis exploratorio sobre la distribución de los índices profundidad de sondaje y nivel de inserción, utilizando medidas de tendencia central y de dispersión. Se obtuvieron frecuencias y proporciones de cada uno de los microorganismos estudiados. Para las variables hábito de fumar, placa bacteriana, sangrado al sondaje y compromiso de furcación se calcularon frecuencias y proporciones. Un valor $\mathrm{p}<0,05$ se estableció como el nivel de significancia estadística para todas las pruebas.

Mediante un análisis de regresión multinivel se evaluó la influencia de diferentes factores sobre la profundidad de sondaje. Se realizó un modelo de regresión multinivel con intercepto aleatorio en tres niveles: localización (nivel 1), diente (nivel 2) y sujeto (nivel 3). 
Se efectuó un modelo de componentes de la varianza (modelo vacío) utilizando la profundidad de sondaje como variable dependiente pero sin incluir variables explicativas. El modelo vacío se usó para estimar la variabilidad total de la profundidad de sondaje atribuida al nivel paciente, diente o localización. Posteriormente se incluyeron en el modelo una serie de variables explicativas con el fin de establecer la asociación entre cada covariable y la variable dependiente. Se calculó el cambio de ajuste de cada modelo (-2log likehood) incluyendo/excluyendo variables explicativas y se evaluó la significancia estadística mediante pruebas de chi cuadrado.

Se utilizó un programa estadístico para todos los análisis (SPSS, versión 15, Chicago, IL) y se usó un programa diseñado específicamente para análisis multinivel (Multilevel Models Project institute of education. Mlwin, version 2.19, Londres, Reino Unido).

\section{RESULTADOS}

Se estudiaron 31 hombres (41\%) y 45 mujeres (59\%) con periodontitis crónica. La Tabla l describe el nivel al que pertenece cada una de las variables estudiadas. Un total de 188 colonias fueron completamente identificadas en los 76 pacientes estudiados. Los microorganismos más prevalentes fueron $P$. gingivalis $(64,4 \%), F$. nucleatum $(46,3 \%)$ y $P$. intermedia/nigrescens $(44,3 \%)$ (Tabla 2). Se encontraron bacilos entéricos gram negativos en $20(26,31 \%)$ de 76 pacientes. Se observaron especies Enterobacteriaceae en $16(21,05 \%)$ y Pseudomonas aeruginosa en $4(5,26 \%)$ de 20 aislamientos. Se encontró Klebsiella pneumoniae en 12 pacientes y Serratia marcescens en dos pacientes. Se identificaron también otras dos especies de Enterobacteriaceae. La prevalencia de levaduras en las bolsas periodontales de pacientes con periodontitis crónica fue de $13,2 \%$ (10/76). Se encontraron ocho sujetos con Candida albicans y dos pacientes con especies de Candida no específicas. En el modelo multinivel la presencia de microorganismos no fue significativa.

En el análisis multinivel se incluyeron 1.870 dientes y 11.220 sitios. En la Tabla 3 se presentan los resultados del modelo multinivel incluyendo la profundidad de sondaje como variable dependiente y las variables explicativas estadísticamente significativas. En el modelo vacío se obtuvo la variabilidad de cada nivel individual calculando el porcentaje de la variabilidad total adicionando todos los estimadores simultáneamente. La mayor parte de la varianza se atribuyó al

\begin{tabular}{|c|c|}
\hline \multicolumn{2}{|c|}{$\begin{array}{c}\text { TABLA 1.- CARACTERÍSTICAS } \\
\text { CLÍNICASY DEMOGRÁFICANS DE LOS } \\
\text { INDIVIDUOS EN LOS TRES NIVELES } \\
\text { JERÁRQUICOS }\end{array}$} \\
\hline Parán & Valor \\
\hline $\begin{array}{l}\text { VARIABLES DEL NIVEL } 3 \text { (PACIENTE) } \\
\text { - Edad (promedio } \pm D E \text { ) } \\
\text { - Sexo femenino } \\
\text { - Fumadores } \\
\text { - Presencia de entéricos } \\
\text { - Presencia de Aa } \\
\text { - Presencia de Pg } \\
\text { - Presencia de Tf } \\
\text { VARIABLES DEL NIVEL } 2 \text { (DIENTE) } \\
\text { - Compromiso de furcación } \\
\text { VARIABLES DEL NIVEL } 1 \text { (LOCALIZACIÓN) } \\
\text { - Profundidad sondaje (promedio } \pm S D \text { ) } \\
\text { - Nivel inserción clínica (promedio } \pm S D) \\
\text { - Sangrado al sondaje (\%) } \\
\text { - Presencia de placa (\%) }\end{array}$ & $\begin{array}{l}n=76 \\
46 \pm 8 \\
45 \\
(59,2 \%) \\
17 \\
(22,3 \%) \\
20 \\
18 \\
51 \\
43 \\
n=1.870 \\
59 \% \\
n=11.220 \\
5,15 \pm 0,6 \\
5,25 \pm 0,6 \\
73 \% \\
59 \%\end{array}$ \\
\hline \multicolumn{2}{|l|}{$\begin{array}{l}\text { DE: Desviación estándar. } \\
\text { Aa: Aggregatibacter actinomycetemcomitans. } \\
\text { Pg: Porphyromonas gingivalis. } \\
\text { Tf: Tannerella forsythia. }\end{array}$} \\
\hline
\end{tabular}

nivel localización (63\%), seguido por el nivel diente $(23 \%)$ y el nivel paciente (14\%).

La tabla 4 presenta la estimación de la regresión para las covariables estadísticamente significativas. Los sujetos fumadores $(p=0,003)$ se asociaron significativamente con bolsas profundas. En el modelo ninguna otra variable fue significativa en el nivel sujeto.

En el nivel diente, los dientes anteriores presentaron menor profundidad de sondaje $(p=0,0002)$. Por otra parte, los compromisos de furcación no presentaron un impacto significativo sobre la profundidad de sondaje.

En el nivel localización del diente, el modeló presentó una mayor profundidad de sondaje asociada a: locali- 


\begin{tabular}{|c|c|}
\hline \multicolumn{2}{|c|}{$\begin{array}{l}\text { TABLA 2.- PREVALENCIA DE } \\
\text { MICROORGANISIMOS EN } 76 \text { PACIENTES } \\
\text { CON PERIODONTITIS CRÓNICA }\end{array}$} \\
\hline Microorganismo & $\begin{array}{c}\text { Prevalencia } \\
(\%)\end{array}$ \\
\hline Porphyromonas gingivalis & 64,4 \\
\hline Fusobacterium nucleatum & 46,3 \\
\hline Prevotella intermedia/nigrescens & 44,2 \\
\hline Bacilos entéricos gram negativos & 26,3 \\
\hline $\begin{array}{l}\text { Aggregatibacter } \\
\quad \text { actinomycetemcomitans }\end{array}$ & 23,7 \\
\hline Prevotella melaninogénica & 14,2 \\
\hline Levaduras & 13,2 \\
\hline Eikenella Corrodens & 6,5 \\
\hline Tanerella forsythia & 3,9 \\
\hline
\end{tabular}

\section{TABLA 3.- MODELO DE REGRESIÓN LINEAL MULTINIVEL ESTIMANDO LA RELATIVA CONTRIBUCIÓN DE LOS PARÁMETROS A NIVEL SUJETO, DIENTE Y LOCALIZACIÓN EN LA VARIABILIDAD DE LA PROFUNDIDAD DEL SONDAJE}

\begin{tabular}{|l|l|}
\hline Intercepto & $\begin{array}{l}\text { Modelo vacío } \\
\text { B } \pm \text { DE }\end{array}$ \\
\hline & $2,958 \pm 0,081$ \\
Varianza & \\
- Sujeto (nivel 3) & $0,456 \pm 0,082$ \\
- Diente (nivel 2) & $0,839 \pm 0,039$ \\
- Localización (nivel 1) & $2,010 \pm 0,029$ \\
Total varianza & 3,305 \\
\% total varianza & \\
- Sujeto (nivel 3) & 14 \\
- Diente (nivel 2) & 23 \\
- Localización (nivel 1) & 63 \\
- -2 LL & $42.199,666$ \\
\hline DE: Desviación estándar. \\
\hline
\end{tabular}

\section{TABLA 4.- MODELO DE REGRESIÓN LINEAL IMULTINIVEL EVALUANDO LA SIGNIFICANCIA DE LOS PARÁIMETROS EN LOS TRES NIVELES Y EXPLICANDO LA VARIABILIDAD EN LA PROFUNDIDAD DEL SONDAJE}

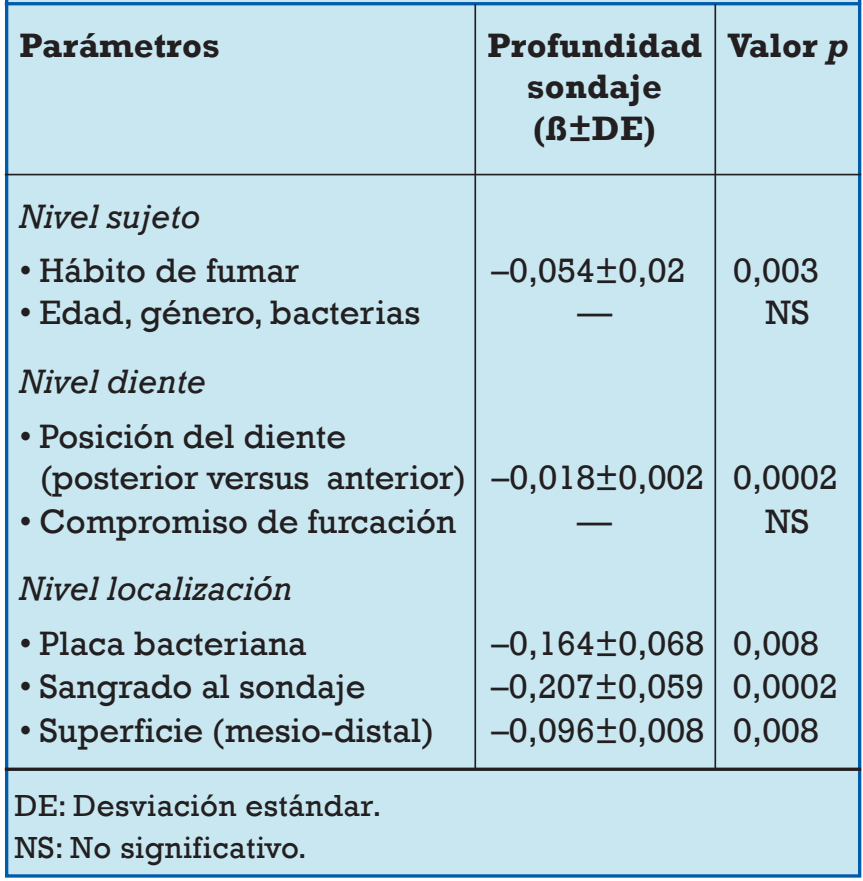

zaciones proximales $(p=0,008)$, sangrado al sondaje $(p=0,0002)$ y presencia de placa bacteriana $(p=0,008)$.

\section{DISCUSIÓN}

En el presente estudio se utilizó un modelo de regresión múltiple multinivel para analizar la influencia jerárquica de los niveles sujeto, diente y localización sobre la profundidad de sondaje de 76 pacientes con periodontitis crónica. La mayor parte de la varianza se atribuyó al nivel localización (63\%) seguido por el diente $(23 \%)$ y el nivel paciente (14\%). Estos resultados implican que la mayor variación en la profundidad de sondaje se debe a factores que actúan a nivel localización en el diente. Estas observaciones corroboran los resultados de estudios previos donde se observó que los factores a nivel localización presentaron un mayor impacto que las variables a nivel sujeto cuando evaluaron la relativa contribución de la variabilidad multinivel (10-14). 
El microorganismo mas prevalente en este estudio fue P. gingivalis $(64,4 \%)$, frecuencia similar a la de un estudio realizado recientemente en Colombia (15). Los niveles de $A$. actinomycetemcomitans observados en pacientes con periodontitis crónica difieren entre las poblaciones estudiadas y su prevalencia varía entre el $8 \%$ y el $57 \%$ en Norteamericanos, Europeos y Asiáticos (16-18). Los bacilos entéricos gram negativos encontrados en este estudio $(26,31 \%)$ presentaron una prevalencia similar a la informada por otros investigadores en Colombia $(15,19)$ y Brasil (20). La prevalencia de levaduras en esta investigación (13,2\%) fue similar a la encontrada por Slots y colaboradores (21) quienes registraron $13-14 \%$ de levaduras en bolsas periodontales de pacientes con periodontitis refractaria. Es importante tener en cuenta que la presencia de periodontopatógenos, bacilos entéricos gram negativos y Candida no fueron significativos en el modelo multinivel (Tabla 4). Este hallazgo confirma los resultados observados por D'Aiuto y colaboradores (11) quienes evaluaron mediante un análisis multinivel la contribución de los periodontopatógenos sobre la variabilidad de los resultados clínicos después de la terapia mecánica periodontal.

Los resultados de la presente investigación asociaron una mayor profundidad de sondaje con variables del sujeto (hábito de fumar), del diente (tipo de diente) y de localización en el diente (localizaciones proximales, presencia de placa y sangrado al sondaje), confirmando la evidencia que indica como las mediciones de la profundidad de sondaje en diferentes localizaciones y dientes del mismo paciente no son independientes (10-14).

El análisis de los resultados reveló una mayor profundidad de sondaje en los dientes posteriores comparado con los dientes anteriores. Otros investigadores encontraron resultados similares empleando modelos multinivel (9). En este sentido es importante tener presente que los dientes anteriores cicatrizan con mayor recesión y presentan mayor reducción de la bolsa periodontal que los dientes posteriores $(11,12)$. En la presente investigación, el compromiso de furcación no presentó un impacto significativo sobre la profundidad de sondaje como lo demostraron recientemente dos estudios multinivel, debido principalmente a que esta variable fue analizada en el nivel diente y no en el nivel localización $(10,14)$. Por otra parte, la movilidad dental no se incluyó en el presente estudio debido a que esta variable presentó resultados divergentes en algunos estudios que utilizaron análisis multinivel (11).

En el nivel localización, se observó mayor profundidad de sondaje en localizaciones interproximales comparado con localizaciones bucales y linguales. Estos resultados corroboran los obtenidos por una publicación previa (22) y son consistentes con el conocimiento actual en donde se reconoce una mayor profundidad de sondaje a nivel proximal (11). En esta investigación, la presencia de placa bacteriana y sangrado se asoció con localizaciones con mayor profundidad al sondaje, confirmando los resultados informados en publicaciones recientes $(11,22)$. Los hallazgos de la presente investigación enfatizan la importancia de tener presente los factores relacionados con la localización en el diente sobre la planificación de una terapia apropiada, para aquellas localizaciones asociadas con una mayor profundidad al sondaje, sangrado y presencia de placa. Este enfoque también está de acuerdo con las recomendaciones de reconocidas agremiaciones científicas que sugieren una evaluación jerárquica de la enfermedad periodontal, teniendo en cuenta las características particulares del paciente (23).

\section{CONCLUSIONES}

En el presente estudio la mayor parte de la varianza se atribuyó al nivel localización implicando una mayor variabilidad en la profundidad de sondaje debido a factores que actúan a ese nivel. El modelo multinivel es un modelo analítico promisorio que por su contribución al análisis de los datos debe considerarse en el diseño de estudios epidemiológicos. Posteriores investigaciones en periodoncia deben enfocarse en el análisis de la influencia de variables relacionadas con el nivel localización del diente debido a sus implicaciones terapéuticas.

\section{BIBLIOGRAFÍA}

1. Ledder RG, Gilbert P, Huws SA, Arons L, Ashley MP, Hull PS, et al. Molecular analysis of the subgingival microbiota in health and disease. Appl Environ Microbiol 2007;73:516-23.

2. Gemmell E, Seymour GJ. Immunoregulatory control of Th1 / Th2 cytokine profiles in periodontal disease. Periodontol 2000 2004;35:21-41.

3. Armitage GC, Cullinan MP. Comparison of the clinical features of chronic and aggressive periodontitis. Periodontology 2000 2010;53:12-27.

4. Axtelius B, SoÈderfeldt B, AttstroÈm R: A multilevel analysis of factors affecting pocket probing depth in patients 
responding differently to periodontal treatment. J Clin Periodontol 1999;26:67-76.

5. Offenbacher S. Periodontal diseases: pathogenesis. Ann Periodontol 1996;1:821-78.

6. Armitage GC. Development of a classification system for periodontal diseases and conditions. Ann Periodontol 1999;4:1-6.

7. Hamp SE, Nyman S, Lindhe J. Periodontal treatment of multirooted teeth. Results after 5 years. J Clin Periodontol 1975;2:126-35.

8. Slots J. Rapid identification of important periodontal microorganisms by cultivation. Oral Microbiol Immunol 1986; 1:48-55.

9. Tomasi C, Koutouzis T, Wennström JL. Locally delivered doxycycline as an adjunct to mechanical debridement at retreatment of periodontal pockets. J Periodontol 2008;79:431-9.

10. D'Aiuto F, Ready D, Parkar M, Tonetti MS. Relative contribution of patient-, tooth-, and site-associated variability on the clinical outcomes of subgingival debridement. I. Probing depths. J Periodontol 2005;76: 398-405.

11. Tomasi C, Leyland AH,Wennström JL. Factors influencing the outcome of non-surgical periodontal treatment: a multilevel approach. J Clin Periodontol 2007;34: 682-90.

12. Axtelius B, SoÈderfeldt B, Attstroł̀m R: A multilevel analysis of factors affecting pocket probing depth in patients responding differently to periodontal treatment. J Clin Periodontol 1999;26:67-76.

13. Dannewitz B, Lippert K, Lang NP, Tonetti MS, Eickholz P. Supportive periodontal therapy of furcation sites: nonsurgical instrumentation with or without topical doxycycline. J Clin Periodontol 2009;36:514-22.

14. Haffajee AD, Torresyap G, Socransky SS. Clinical changes following four different periodontal therapies for the treatment of chronic periodontitis: 1 year results. J Clin Periodontol 2007;34:243-53.

15. Lafaurie GI, Contreras A, Baron A, Botero J, MayorgaFayad I, Jaramillo A et al. Demographic, clinical, and microbial aspects of chronic and aggressive periodontitis in Colombia: a multicenter study.J Periodontol 2007; 78:629-39.
16. Riggio MP, Macfarlane TW, Mackenzie D, Lennon A, Smith AJ, Kinane D. Comparison of polymerase chain reaction and culture methods for detection of Actinobacillus actinomycetemcomitans and Porphyromonas gingivalis in subgingival plaque samples. J Periodontal Res 1996;31:496-501.

17. Lau L, Sanz M, Herrera D, Morillo JM, Martín C, Silva A. Quantitative real-time polymerase chain reaction versus culture: a comparison between two methods for the detection and quantification of Actinobacillus actinomycetemcomitans, Porphyromonas gingivalis and Tannerella forsythensis in subgingival plaque samples. J Clin Periodontol 2004;31:1061-9.

18. Wu YM, Yan J, Chen LL, Gu ZY. Association between infection of different strains of Porphyromonas gingivalis and Actinobacillus actinomycetemcomitans in subgingival plaque and clinical parameters in chronic periodontitis. J Zhejiang Univ Sci B. 2007;8:121-31.

19. Botero JE, Arce RM, Escudero M, Betancourth M, Jaramillo A, Contreras Al. Ocurrence of periodontopatic and superinfecting bacteria in chronic an aggressive periodontitis subject in a Colombia population. J Periodontol. 2007;78:696-704.

20. Barbosa FCB, Mayer MPA, Saba-Chuifi E, Cai S. Subgingival occurrence and antimicrobial susceptibility of enteric rods and pseudomonads from Brazilian periodontitis patients. Oral Microbiol Immunol. 2001;16:306-10.

21. Slots J, Feik D, Rams TE. Age and sex relationships of superinfecting microorganisms in periodontitis patients. Oral Microbiol Immunol 1990;5:305-8.

22. Haffajee AD, Torresyap G, Socransky SS. Clinical changes following four different periodontal therapies for the treatment of chronic periodontitis: 1 year results. J Clin Periodontol 2007; 34:243-53.

23. Armitage GC, Offenbacher S. Consensus report on periodontal diseases: epidemiology and diagnosis. Ann Periodontol 1996;1:216-22.

\section{CORRESPONDENCIA}

\author{
Carlos M. Ardila M \\ Calle $64 \mathrm{~N}^{\circ}$ 52-59 \\ Medellín. Colombia \\ e-mail: martinardila@gmail.com
}

